\title{
Essais de distributions discontinues de phosphates naturels dans l'alimentation des bovins tropicaux ${ }^{(*)}$
}

\author{
par H. SERRES (**) et L. BERTAUDIÈRE (***) \\ (avec la collaboration technique de $\mathbf{M}^{\text {me }}$ IENNY)
}

\begin{abstract}
RÉSUMÉ
Les auteurs ont envisagé l'emploi de phosphate naturel en dépit de sa teneur en fluor pour la complémentation phosphocalcique, économique, du bétail tropical en utilisant des distributions discontinues capables de permettre une élimination du fluor qu'il contient.

Une expérimentation a donc été faite sur soixante taurillons zébus au Tchad. Annuellement et à trois reprises, ils ont reçu pendant une période limitée $50 \mathrm{~g}$ de phosphate naturel par tête et par jour. Une telle administration provoque des lésions du squelette et des dents en raison de la terleur en fluor du phosphate. Les lésions dentaires ne sont graves que dans une minorité des cas, mais elles ne régressent pas. En revanche les lésions du squelette et des articulations peuvent être importantes et provoquer des troubles fonctionnels (boiteries), mais après quelques mois sans phosphate, elles régressent de façon quasi totale. Au cours de l'essai aucun animal n'a succombé à la fluorose et les animaux qui ont terminé l'expérience étaient en très bon état. Les dosages de fluor dans le squelette montrent que son taux s'élève fortement pendant les périodes d'administration de phosphate mais qu'il diminue entre ces périodes, par suite d'une élimination jusqu'à un taux compatible avec une structure normale de l'os, alors que la teneur des tissus mous et du lait reste négligeable.
\end{abstract}

Dans les régions intertropicales à longue saison sèche et arides, d'immenses troupeaux de bovins sont élevés, selon un mode très extensif. Ils subissent chaque année une sous-alimentation grave, particulièrement au cours de la deuxième moitié de la saison sèche. Energie, matières azotées, minéraux, vitamines sont alors insuffisants dans la ration. Les corrections en énergie et matières azotées sont sans aucun doute les plus efficaces. Mais leur coût, en éle-

(*) Ce travail a été réalisé grâce au soutien financier de l'Institut Mondial du Phosphate, 8, rue de Penthièvre, 75008 Paris.

Un document plus complet concernant l'expérimentation est disponible auprès des auteurs et de l'IMPHOS.

(**) I. E. M. V. T., 10, rue Pierre-Curie, 94700 Maisons-Alfort (France).

(***) Laboratoire de Farcha, N'Djamena, Tchad. vage extensif de zone aride, peut les rendre non économiques. A Dakar, CALVET et collab. (1) ont montré qu'un complément minéral phosphaté était capable d'être, en fonction de son coût, le plus efficace. Dans de nombreux pays tropicaux, on trouve des gisements de phosphates constitués d'apatites dont la base est le phosphate tricalcique, capable d'apporter du phosphore et du calcium. Selon GUEGUEN (2), leur utilisation digestive peut atteindre 50 p. 100 surtout par les animaux carencés qui ont un pouvoir de fixation plus élevé. Malheureusement, les apatites des gisements naturels contiennent du fluor à des taux qui avoisinent 3 p. 100 . Le fluor, à faible concentration est un constituant normal de l'organisme et se révèle favorable à la lutte contre les caries dentaires. Mais à forte concentration, il provoque des lésions des os et 
des incisives que VELU (7) a décrites sous le terme de "Darmous». De nombreuses études ont été consacrées à l'intoxication fluorée consécutive à l'absorption continue de fluor soit par la contamination des aliments auprès des industries $\mathrm{du}$ phosphate, soit par la boisson d'eaux fluorées. Ces études ont montré que le fluor se stocke spécifiquement dans le squelette et dans les dents, alors que les muscles, les viscères, le lait n'en contiennent pratiquement pas, ce qui évite les problèmes de santé publique. Plusieurs travaux ont aussi démontré que la fluorose n'affecte ni la croissance, ni la fertilité, ni la production laitière, sauf dans les cas où de très graves lésions du squelette entraînent des troubles fonctionnels.

Mais lorsque l'administration de fluor dure un temps limité, on constate que ce dernier s'élimine par l'urine après l'arrêt de l'absorption. LARGENT (3) avait déjà observé le phénomène sur lui-même. SUTTIE (6) l'a vérifié également sur des vaches laitières.

D'où l'idée de tenter de corriger, à faible coût, les carences phosphocalciques périodiques $\mathrm{du}$ bétail tropical par des distributions de phosphate naturel de durée limitée. Afin de vérifier l'exactitude de l'hypothèse d'une élimination $\mathrm{du}$ fluor, nous avons choisi d'en distribuer des doses fortes pour faire apparaître sûrement des lésions squelettiques et dentaires et d'en observer l'évolution.

\section{MATÉRIEL ET MÉTHODES}

\section{Les animaux}

Nous avons utilisé des zébus de race locale au Tchad, dits zébus arabes ou «Choa ». Au début de l'essai les animaux, des mâles, avaient environ deux ans.

Ils ont été débarrassés de leurs parasites externes et internes, puis vaccinés contre les maladies contagieuses régionales. Leur régime alimentaire durant l'expérimentation était le plus proche possible de celui que les bovins ont à l'ordinaire dans ce pays : pâturage naturel pendant la période sans phosphate, foin de graminées naturelles pendant les périodes de distribution.

L'expérimentation a porté sur soixante animaux. Pour des raisons de sécurité du protocole, soixante-cinq têtes ont été mises en expérience au début de l'essai. Les animaux en surnombre ont été éliminés progressivement lorsque leur maintien a été jugé inutile.

\section{Le phosphate naturel utilisé provient du Togo}

Il est simplement finement broyé. Il contient en moyenne 12 p. 100 de phosphore et 3,5 p. 100 de fluor.

La distribution quotidienne sera de $50 \mathrm{~g}$, soit $6 \mathrm{~g}$ de phosphore et $1,6 \mathrm{~g}$ de fluor, ce qui représente une dose forte. Le phosphate naturel est distribué individuellement chaque matin à la sortie de l'étable, les $50 \mathrm{~g}$ suspendus dans environ $200 \mathrm{ml}$ d'eau et administrés à la bouteille. Les animaux s'habituent très vite à cette pratique et il n'y a pas de pertes.

\section{Le protocole expérimental}

A l'origine de l'expérimentation il était prévu 4 mois d'administration quotidienne de phosphate, de mars à juin en fin de saison sèche. C'est ce qui a été réalisé la première année. Mais en raison de la fréquence et de l'importance des lésions observées, l'administration a été réduite à 3 mois en deuxième année et à. 80 jours la troisième.

L'expérimentation comporte donc en alternance trois phases de distribution et deux de déstockage de 8 et 9 mois.

Les contrôles expérimentaux ont été les suivants :

- Examen de l'état clinique des animaux, particulièrement en ce qui concerne les os explorables et les articulations, mais aussi tout autre trouble de la santé ;

- Examen des dents et surveillance de l'apparition des lésions dentaires; celles-ci sont notées selon les conventions indiquées par G. MLLHAUD et J. C. GODFRAIN (5) :

\section{Dent normale}

1 Effet douteux

2 Effet léger : opacification légère de l'émail

3 Effet modéré : opacification forte de l'émail, crayeux et strié ;

4 Effet marqué : émail crayeux, érodé, portant de petites taches brunes;

5 Effet excessif : larges érosions de l'émail, grandes taches noires, ușure excessive de la dent.

- Dosages du fluor dans les os maxillaires et métacarpiens principaux sur des lots de dix animaux abattus à chaque phase de l'expérience, juste avant et à la fin de chaque distribution de phosphate. 
Le dosage du fluor est fait sur les cendres de chacun des os prélevés. On utilise une technique ionométrique à l'aide d'une sonde spécifique ORION selon la méthode de MAUZAC et collab. (4). En utilisant un tampon au citrate de sodium, la limite de sensibilité se situe aux environs de 0,1 p. p. m. en fluorure de sodium avec une bonne reproductibilité. Les résultats seront finalement exprimés en p. p. m. de fluor dans les cendres des os.

\section{RÉSULTATS EXPÉRIMENTAUX}

\section{A. Etude clinique des animaux}

a) Etude des lésions squelettiques des animaux

\section{Première administration de phosphate}

Pendant les 10 premières semaines d'administration de phosphate, aucune anomalie n'a pu être décelée. Les premières ont été observées à la fin du $3^{\mathfrak{e}}$ mois d'administration. Ce sont essentiellement des exostoses qui siègent sur les os canons et les têtes articulaires au niveau de l'épaule (humérus, radius) et du grasset (fémurtibia).

D'abord décelables à la palpation, elles deviennent visibles par la déformation qu'elles provoquent en se développant entre le $90^{\mathrm{e}}$ et le $120^{\circ}$ jour d'administration de phosphate.

Suivant leur localisation, elles entraînent ou non des boiteries. Des exostoses existent aussi au niveau des maxillaires et des côtes, provoquant des déformations, mais sans troubles fonctionnels associés.

A l'issue de la première période d'administration du phosphate, sur cinquante-trols animaux (trois animaux en réserve), quarantehuit présentaient des lésions squelettiques dont la distribution est donnée au tableau récapitulatif $n^{0} \quad 1$.

Une majorité de ces lésions étaient importantes, ce qui est attesté par huit cas de parésie et seize cas de boiteries dont plusieurs très fortes.

\section{Première période sans phosphate}

Les taurillons ayant été reconduits sur les pâturages naturels, progressivement les troubles fonctionnels se sont atténués pour disparaître complètement dans la plupart des cas. Les taurillons ont pris du poids et grandi.

Après 6 mois, les lésions ne sont plus observables à l'inspection. Sur un certain nombre d'animaux, les séquelles des lésions antérieures sont décelables à la palpation attentive des lieux où elles avaient été repérées. Ce sont sur les métacarpiens et les métatarsiens, faciles à explorer, que l'on peut le plus facilement les identifier par une irrégularité de la surface osseuse, sous la peau (voir tableau récapitulatif $n^{*} 1$ ).

Seuls trois animaux boitent très légèrement lorsqu'on les fait courir, sans que cette observation puisse être rapportée avec certitude à une fluorose.

\section{Deuxième administration de phosphate}

Elle est pratiquée sur trente-deux animaux. Comme en première période, on observe d'abord une phase silencieuse, au cours de laquelle aucun trouble n'apparaît. Mais après 2 mois $1 / 2$, on décèle les premières exostoses et boiteries.

Les symptômes de fluorose squelettique apparaissent donc un peu plus tôt que lors de la distribution précédente, ce qui détermine à arrêter l'administration de phosphate au bout de 3 mois. Le tableau récapitulatif permet de voir comment se distribuent les lésions.

Par rapport aux observations de 1976, on remarque une fréquence plus faible des lésions sur les rayons osseux supérieurs des membres (scapulum, humérus, fémur, tibia) ainsi que sur les rayons inférieurs (phalanges).

Les lésions des rayons moyens : métacarpiens et métatarsiens principaux (os canons) restent nombreuses.

En conséquence, les arthrodystrophies de l'épaule, du coude, du grasset, du jarret sont rares, mais des boiteries légères sont néanmoins observées.

\section{Deuxième période sans phosphate}

Au cours de la deuxième période sans phosphate qui a duré 9 mois, les lésions ont guér1 pratiquement en totalité. Sur vingt têtes, deux seulement présentaient des séquelles légères d'exostoses des métatarsiens, décelables à une soigneuse palpation et, au mois de mars 1978, un seul animal feignait une boiterie.

\section{Troisième distribution de phosphate}

Au cours de la troisième distribution de phosphate, sur les dix animaux restant, la situation n'a que peu évolué en 80 jours. Deux animaux présentaient des exostoses costales et deux des lésions des métatarsiens. 
TABLEAU $\mathrm{N}^{\circ} \mathrm{I}$ - Evolution c1inigue - Squelette

\begin{tabular}{|c|c|c|c|c|c|}
\hline \multirow[b]{4}{*}{ Osteodystrophies } & \multirow{2}{*}{$\frac{1976}{\begin{array}{c}\text { Après } 120 \\
\text { jours de } \\
\text { drogage }\end{array}}$} & \multicolumn{2}{|r|}{1977} & \multicolumn{2}{|c|}{1978} \\
\hline & & $\begin{array}{l}\text { Après } 9 \mathrm{~m} \text { de } \\
\text { destockage }\end{array}$ & $\begin{array}{c}\text { Après } 9 \text { mois de } \\
\text { destockage }+ \\
90 \mathrm{j} \text { de drogage }\end{array}$ & $\begin{array}{l}\text { Après } 9 \mathrm{~m} \mathrm{de} \\
\text { destockage }\end{array}$ & $\begin{array}{l}\text { Après } 9 \text { mois de } \\
\text { destockage }+ \\
80 \mathrm{j} \text { de drogage }\end{array}$ \\
\hline & $N: 53$ & 42 & 32 & 20 & 10 \\
\hline & & & & & \\
\hline -Ligne dorso-vertêbrale & 5 & 0 & 2 & 0 & 0 \\
\hline -Paroi costale & 6 & 0 & 0 & 0 & 2 \\
\hline -Métacarpiens & 37 & 6 & 6 & 0 & 0 \\
\hline -Métatarsiens & 33 & 6 & 7 & 0 & 2 \\
\hline $\begin{array}{l}\text {-Mëtacarpiens + mëtatarsiens } \\
\text { associës }\end{array}$ & 29 & 6 & 17 & 2 & 0 \\
\hline -Maxillaires (inf. et sup.) & 8 & 0 & 10 & 0 & 0 \\
\hline -Scapulum & 10 & 0 & 0 & 0 & 0 \\
\hline -Radius-cubitus & 0 & 0 & 0 & 0 & 0 \\
\hline -Humërus & 25 & 0 & 1 & 0 & 0 \\
\hline -Tibia & 7 & 1 & 1 & 0 & 0 \\
\hline -Fémur & 2 & 0 & 0 & 0 & 0 \\
\hline -Phalanges antêrieures & 10 & 1 & 0 & 0 & 0 \\
\hline -Phalanges postêrieures & 7 & 0 & 1 & 0 & 0 \\
\hline Arthrodystrophies & & & & & \\
\hline -Art, scapulo-humëral & 10 & 2 & 0 & 0 & 0 \\
\hline -Art, humero-radial & 8 & 7 & 2 & 0 & 0 \\
\hline -Art. du carpe & 6 & 5 & 0 & o & 0 \\
\hline -Art. métacarpo-phalangienne & 3 & 3 & 1 & 0 & o \\
\hline -Art. femoro-tibial & 1 & 0 & 0 & 0 & 0 \\
\hline -Art. du tarse & 10 & 8 & 0 & 0 & 0 \\
\hline $\begin{array}{l}\text {-Art, metatarso-phalangientse } \\
\text { Trouble de la locomotion }\end{array}$ & 4 & 3 & 0 & 0 & 0 \\
\hline -Parésie & 8 & 3 & 5 & 0 & 0 \\
\hline -Boiterie avant & 4 & 1 & 3 & 0 & 0 \\
\hline -Boiterie arrière & 12 & 2 & 5 & 1 & 0 \\
\hline Etat d'entretien & & & & & \\
\hline $\begin{array}{l}\text {-Bon (engraissement suffisant, } \\
\text { graisse de surface) } \\
\text {-Moyen (pas de graisse de } \\
\text { couverture mais bon } \\
\text { développement musculaire } \\
\text {-Médiocre (amyotrophie-tnaigreur }\end{array}$ & $\begin{array}{l}19 \\
34\end{array}$ & $\begin{array}{r}26 \\
3\end{array}$ & $\begin{array}{r}18 \\
8\end{array}$ & $\begin{array}{l}2 \\
0\end{array}$ & $\begin{array}{l}0 \\
0\end{array}$ \\
\hline
\end{tabular}

Mais les choses se limitaient là et, entre autres, aucune boiterie même légère n'était décelable.

On a l'impression que les animaux finissent par s'habituer au fluor dans une sorte de mithridatisme.

\section{b) Etude des lésions dentaires}

Nous rappellerons que les dents de lait ne sont jamais atteintes de lésions de fluorose, pour des raisons qui ne sont d'ailleurs pas clairement élucidées.

Quant aux dents d'adultes, elles n'apparaissent lésées que si leur éruption se produit à un moment où l'animal se trouve sous excès de fluor, pen- dant les périodes d'administration et les semaines qui suivent.

Lorsque les dents sont sorties et arrivées au niveau, il ne s'installe plus de lésions nouvelles.

\section{Première administration de phosphate}

$\mathrm{Au}$ cours de cette première phase expérimentale les animaux, pour la plupart jeunes, n'avaient que peu de dents d'adultes. Sur les animaux qui les ont vu apparaître on n'a remarqué que des lésions très limitées, ne dépassant jamais le stade 1 : « effet douteux, modifications de faible importance ne portant pas atteinte à la qualité de l'émail ». 
Le peu d'importance de ces lésions dentaires contraste avec la sévérité des lésions du squelette que nous avons décrites à la suite de cette première administration.

\section{Première période de déstockage}

Pendant la première partie de cette période, les animaux restent chargés en fluor et l'âge aidant, les dents d'adultes sortent en assez grand nombre. On relève neuf dents fortement atteintes (cotées 4 et 5) sur cent soixante-huit, soit 5,3 p. 100 . Les premières mitoyennes ne sont lésées qu'à deux reprises sur neuf et jamais les pinces.

3. Deuxième période d'administration de phosphate

L'accumulation de fluor reprend et l'évolution des anomalies dentaires se poursuit progressivement. Le nombre de dents fortement atteintes (quatre et cinq) est de seize sur cent soixante, soit 12,5 p. 100 . Sur ces seize dents lésées, on ne trouve que deux premières mitoyennes et aucune pince.

4. Deuxième période sans distribution de phosphate

Une certaine stabilisation semble s'établir. On note quinze lésions importantes sur quatrevingts dents, soit 18,75 p. 100 . Deux premières mitoyennes seulement sont lésées sur les quinze et les pinces restent toutes indemnes.

\section{Dernière distribution de phosphate}

On dénombre six dents lésées aux niveaux 3 et 4 sur quarante, soit 15 p. 100 . Deux premières mitoyennes sont intéressées et toujours aucune pince.

\section{Conclusions}

Le nombre de dents porteuses de lésions nettes augmente au cours de l'expérience, mais reste toujours inférieur à 20 p. 100 .

On remarque avec netteté que les pinces, incisives principales, ne sont jamais atteintes alors qu'elles sont les dents les plus importantes pour la préhension des aliments.

Les premières mitoyennes qui les jouxtent et sont les secondes en importance ne sont lésées qu'avec une fréquence modérée. Ce sont surtout les deuxièmes mitoyennes et les coins qui sont le plus souvent atteints.

Nous ne pouvons pas terminer sans signaler que les molaires ne subissent pratiquement pas l'influence de la surcharge fluorée de l'organisme.

Au bilan, la fluorose se manifeste par des lésions nettes des dents marginales de l'arcade incisive, en respectant le plus souvent les incisives centrales et en laissant intactes les molaires.

En conséquence, si l'on sait que la préhension de l'herbe est faite prioritairement par la langue, et que les molaires seules interviennent dans la rumination, peut-être ne faut-il pas attribuer une importance dramatique aux répercussions dentaires de la fluorose sur la santé des animaux. Sans doute intervient-elle pour accélérer la décrépitude de l'arcade incisive lorsque les anımaux vieillissent et doit-elle devenir plus importante chez les vaches âgées.

B. Dosages du fluor dans les os (p. p. m. dans les cendres)

Les résultats des dosages sont résumés au tableau $\mathrm{n}^{\circ}$ II :

Les graphiques 1 et 2 visualisent l'évolution de la teneur en fluor.

\begin{tabular}{|c|c|c|c|c|c|c|}
\hline & \multirow{2}{*}{ Nombre } & \multicolumn{2}{|c|}{ Saxillaire } & \multicolumn{3}{|c|}{$C a n$} \\
\hline & & Moyenne & Erreur standard & Moyenne & Erreur & c standard \\
\hline Témoins & 10 & 630,8 & 214 & 561,8 & & 173 \\
\hline $\begin{array}{l}\text { Après } 120 \mathrm{j} \\
\text { de phosphare }\end{array}$ & 10 & 4842 & 1718 & 4166 & & 1506 \\
\hline $\begin{array}{l}\text { Après } 240 \mathrm{j} \\
\text { de destockage }\end{array}$ & 10 & 4356 & 1158 & 4711 & & 1307 \\
\hline $\begin{array}{l}\text { Après } 90 \mathrm{j} \\
\text { de phosphate }\end{array}$ & 10 & 12931 & 1374 & 12502 & & 1588 \\
\hline $\begin{array}{l}\text { Après } 270 \mathrm{j} \\
\text { de destockage }\end{array}$ & 10 & 3747,2 & 604 & 3147,6 & & 590 \\
\hline $\begin{array}{l}\text { Après } B O \mathrm{j} \\
\text { de phosphate }\end{array}$ & 10 & 7936,4 & 399 & 8595,4 & & 504 \\
\hline
\end{tabular}



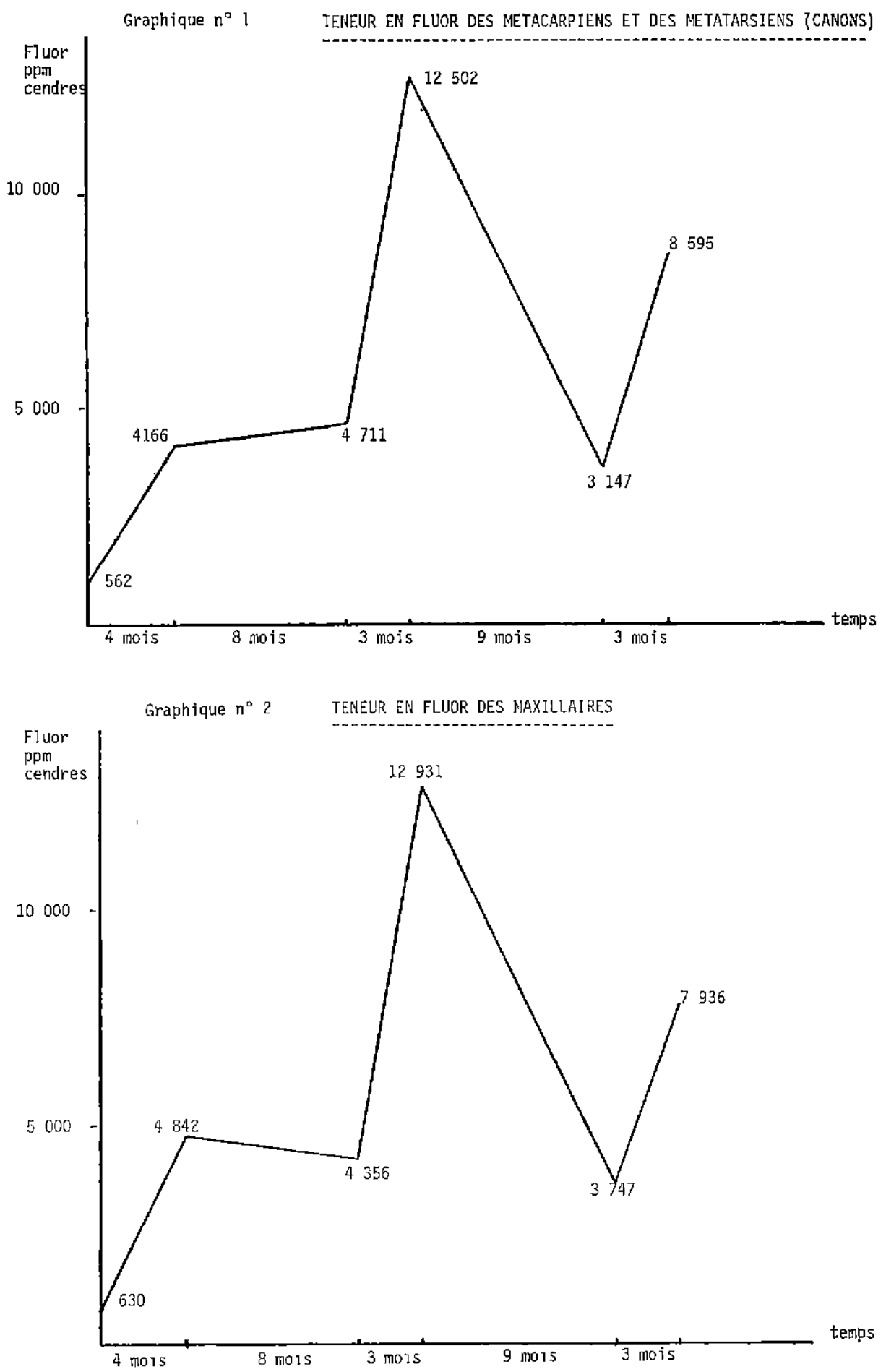

Ces résultats peuvent être commentés de la manière suivante :

a) Similitude des résultats obtenus dans les deux types d'os

Si l'on examine les résultats obtenus en comparant les teneurs en fluor des métacarpiens et des maxillaires ainsi que leur évolution, on est frappé par la ressemblance des courbes qui ne diffèrent que par des détails, en conservant la même forme.

Si l'on veut tenir compte de la précision des mesures, on est amené à admettre que les résultats sont identiques. En effet, chaque moyenne 
pour l'un des deux os se place dans l'intervalle de confiance de l'autre et si l'on effectue des tests de comparaison de moyennes deux à deux pour une même phase expérimentale, on ne trouve aucune différence significative.

\section{b) Interprétation de l'évolution du fluor dans les os}

Au début de l'essai, la teneur des os est inférieure à $1000 \mathrm{ppm}$. On se trouve en effet dans une région où les eaux ne contiennent pas de fluor et où le sol et les plantes n'en sont pas spécialement pourvus.

Le point de départ est donc relativement bas.

- Après la première administration de fluor pendant 4 mois, on observe une forte montée de la teneur des os en fluor aux environs de $4000 \mathrm{ppm}$ pour l'os entier, en même temps qu'apparaissent des lésions.

Mais il est, à ce moment certain que le dosage du fluor total de l'os ne correspond pas à une réalité histologique. En effet, on a dosé le fluor dans plusieurs exostoses qui siègent à la surface et l'on a obtenu les chiffres suivants :

Fluor (ppm)

27324

17035

20274

20024

18507

Moyenne

20358

On observe donc que le fluor est extrêmement concentré dans les lésions superficielles de l'os, ce qui implique une faible pénétration de ce dernier dans les parties profondes, puisque la composition moyenne ne montre pas plus de 4000 ppm.

Cela peut s'expliquer aisément lorsqu'on se rappelle que la croissance en épaisseur se fait à partir du périoste ce qui implique un dépôt plus actif de fluor dans les parties superficielles pendant la période limitée d'administration du phosphate naturel.

- Après 8 mois de régime sans phosphate naturel on observe que la teneur moyenne de l'os entier se situe toujours au niveau de 4000 ppm. La moyenne a très légèrement augmenté dans les métacarpiens et très légèrement diminué dans les maxillaires. Mais compte tenu de l'intervalle de confiance de ces moyennes, ces variations ne sont pas significatives.
Cependant, après la période de régime sans phosphate, les animaux ne présentent plus d'exostoses et on peut penser que le fluor s'est distribué de façon homogène dans le squelette, diminuant ainsi les fortes concentrations superficielles, ce qui permet d'expliquer la récession des lésions observées 8 mois auparavant.

- Après la deuxième administration de phosphate, on voit la teneur fluorée des os monter très rapidement à des concentrations moyennes de 12000 ppm.

L'explication que nous en donnons est la suivante: au début de l'administration, le squelette des animaux se présente morphologiquement d'une façon quasi normale mais biochimiquement il est déjà très chargé en fluor, sans doute à la limite d'une structure normale.

Dès lors, l'administration de fluor se traduit par une montée brutale de son taux dans le squelette avec apparition de lésions visibles sur l'animal vivant et sur les os après l'abattage.

- Après la deuxième période de déstockage on voit que le fluor contenu dans le squelette a beaucoup diminué, pour revenir entre 3000 et $4000 \mathrm{ppm}$. Il est indiscutable qu'une active élimination s'est produite pendant la durée de l'alimentation sans supplémentation en phosphate naturel.

Le fluor accumulé pendant la période de consommation de phosphate a été rejeté jusqu'à l'obtention d'un niveau compatible avec une structure quasi normale des os.

- La troisième distribution de phosphate reproduit dans ses grandes lignes les effets de la deuxième. On observe une vive remontée du taux de fluor au-dessus de $8000 \mathrm{ppm}$, inférieur à celui de l'année précédente car l'administration a été limitée à 80 jours.

On peut regretter de n'avoir pas disposé d'un lot d'animaux plus important de 10 unités, ce qui aurait permis de suivre une période de déstockage supplémentaire et de confirmer l'observation de l'année précédente. Mais au départ de l'expérimentation, nous ignorions quelle allure elle allait prendre et nous souhaitions la limiter à une durée de 2 ans.

\section{c) En conclusion}

Nous pensons que le squelette des zébus peut contenir une teneur en fluor de l'ordre de $3000 \mathrm{ppm}$ (sur les cendres) sans que les signes squelettiques de fluorose soient cliniquement 
décelables. (On trouve d'ailleurs des animaux n'ayant pas consommé de phosphate avec des titres de $1500 \mathrm{ppm}$.)

Au-dessus de ce taux des lésions apparaissent. Mais à l'arrêt de l'administration de phosphate naturel, le fluor s'élimine pour revenir à ce taux de 3000-4000 ppm, qui paraît compatible avec une apparente normalité.

\section{CONCLUSIONS GÉNÉRALES}

A la suite de cette expérimentation, on peut tirer les conclusions suivantes :

1. L'administration de $50 \mathrm{~g}$ de phosphate naturel par jour, qui procure $6 \mathrm{~g}$ de phosphore et $1,6 \mathrm{~g}$ de fluor provoque, si elle est prolongée pendant plus de 3 mois, des symptômes et des lésions de fluorose au niveau du squelette et des dents.

2. Si l'on interrompt la distribution de phosphate naturel pendant une période assez longue, les lésions squelettiques régressent pour devenir difficilement perceptibles après 6 mois.

3. Au cours de l'administration du phosphate naturel au niveau de $50 \mathrm{~g}$ par jour, des lésions dentaires apparaissent au moment de l'éruption des dents d'adulte. Ces lésions sont d'importance variable. Lorsqu'elles sont légères avec conservation de la brillance de l'émail, elles peuvent régresser. Lorsqu'elles sont fortes, elles doivent être considérées comme définitives. Leur fréquence s'accroît avec la répétition des traitements au phosphate naturel. Cependant, la proportion de dents gravement lésées reste relativement peu élevée. Dans le cadre de l'expérimentation que nous avons poursuivie, elle n'a pas eu d'influence sur la façon de se nourrir des animaux qui n'en ont pas été gênés.

Pour des mâles destinés à l'abattage, on peut considérer qu'elle n'est pas très importante. On ne saurait conclure de la même façon pour les reproducteurs dont la vie économique est plus longue et dont les dents devraient être moins durables que les dents normales. Mais cela n'a pas pu être précisé compte tenu de la durée de l'essai.

Il est cependant nécessaire de rappeler que les molaires n'étant pas atteintes, la rumination n'est pas affectée.

4. Les animaux paraissent cliniquement plus sensibles à la fluorose dans leur jeune âge. Il est vrai que, si les doses de phosphate administrées étaient constantes, leur quantité relative par rapport au poids des animaux a décru au cours de l'essai, en raison inverse de la croissance. Il est également certain que les zébus ont des difficultés de croissance sur les pâturages naturels médiocres, pauvres en protéines, tant qu'ils n'ont pas atteint le poids de $200 \mathrm{~kg}$.

Au-dessus de $300 \mathrm{~kg}$, la sensibilité paraît moins forte et les animaux conservent une bonne santé apparente.

5. Le squelette se charge en fluor après la consommation de phosphate naturel.

Au cours d'une première période de consommation de phosphate, il semble que le fluor se distribue irrégulièrement donnant des concentrations plus élevées sous le périoste, ce qui engendre des exostoses.

Par la suite, les taux de fluor peuvent atteindre des niveaux très élevés (12000 ppm), mais alors l'arrêt de consommation de phosphate entraîne une élimination de fluor jusqu'à un niveau compatible avec une structure normale de l'os, qui paraît se situer vers $3000-4000 \mathrm{ppm}$. (Ce qui devrait être vérifié par des expérimentations ultérieures.)

6. Aucune lésion ni symptôme autre que dentaire n'est apparu avant un délai de deux mois de consommation au niveau de $50 \mathrm{~g}$ par jour de phosphate naturel.

En pratique, on devrait pouvoir retenir une complémentation phosphatée à l'aide de phosphate naturel, en la limitant à des périodes de 2 mois au niveau de 30 g/jour. Deux périodes d'administration par an devraient être recommandées.

Une expérimentation permettrait de le vérifier.

\section{SUMMARY}

\section{Discontinuous natural phosphate rock intakes in tropical cattle feeding}

The authors have studied the effect of natural phosphate rock used as an inexpensive calcium phosphate supplementation despite its fluorine content, in tropical cattle, by using discontinuous supplies so as to allow the elimination of the fluorine component. 
Sixty young zebu cattle were part of an experiment carried out in Chad. For 3 years in a row, they were given on a lımited period a time, $50 \mathrm{~g}$ of natural phosphate per head and per day. Such an intake entails skeletal and tooth lesions because of the fluorine content in phosphate. Tooth lesions are not serious in the majority of cases but they don't heal. On the other hand, bone and joint lesions can be severe and entail functional diseases such as limping, but after a few month without phosphate, they heal almost completely. During the experiment no animal died of fluorine poisoning and the animals which came through the experiment were very sound. Fluorine titration in the skeleton shows that the amount of fluorine rises sharply when phosphate is given but it decreases between two intakes as it is eliminated up to a rate compatible with the bone normal structure. Meanwhile the fluorine rate remains very low in soft tissue and milk.

\section{RESUMEN \\ Ensayos de distribuciones discontinuas de fósfato natural en la alimentación de los bovinos tropicales}

Los autores enfocaron el empleo de fósfato natural, a pesar de su proporción de flúor, para la adición fosfocálcica, económica, de las raciones destinadas al ganado tropical, al utilizar distribuciones discontinuas capaces de permitir una eliminación del flúor que contiene.

Pués se hizo una experimentación con 60 novillos cebus en Chad. Recibieron anualmente y tres veces durante un periodo limitado $50 \mathrm{~g}$ de fósfato natural por cabeza y por dia. Tal adminıstración provoca lesiones del esqueleto y de las dientes a causa de la proporción de flúor del fósfato.

Las lesiones dentarias no son graves más que en una minoria de los casos, pero no retroceden. En cambio, las lesiones del esqueleto y de las articulaciones pueden ser importantes y provocar trastornos funcionales (cojeras), pero después de algunos meses sin administración de fósfato, retroceden de modo casi total. Durante el ensayo, ningún animal murió de fluorosis y los animales restantes al fin de la experiencia estaban en muy buen estado. Las dosificaciones del flúor en el esqueleto muestran que su tasa se alza fuertemente durante los periodos de administración de fósfato pero que disminuye entre dichos periodos a consecuencia de una eliminación hasta una tasa compatible con una estrúctura normal del hueso, mientras que la proporción en los tejidos blandos у ел la leche queda despreciable.

\section{BIBLIOGRAPHIE}

1. CALVET (H.), FRIOT (D.), GUEYE (I. S.). Supplémentations minérales, alimenta1res et pertes de poids des zébus sahéliens en saison sèche. Rev. Elev. Méd. vét. Pays trop., 1976, 29 (1) : 59-66.

2. GUEGUEN (L.). Les critères de qualité nutritionnelle des compléments minéraux en alimentation animale. Alim. Vie, 1970, 58 (7-8-9) : 115-129.

3. LARGENT (E. J.) in : MUHLER (J. C.), HINE (M. K.), ed. Fluorine and dental health Bloomugton, Indiana University press, pp. 132.

4. MAUZAC (M.), GUERARD (F.), MATHIEU (J ), LAROCHE (J.). Dosages du fluor et de l'ammo- niaque par électrodes spécifiques. Analusis, 1976 , 4 (7) : 326-329.

5. MILHAUD (G.), GODFRAIN (J. C.). La fluorose bovine d'origine industrielle. Rec. Méd. vét. Alfort, 1975, $191(5)$ : 265-272.

6. SUTTIE (J. W.), CARLSON (J. R.), FALTIN (E. C.). Effects of alterning periods of high and low fluoride ingestion on dairy cattle. $J$. dairy Sci., 1972, 55 (6) : 790-804.

7. VELU (H.). « Le Darmous ». Arch. Inst. Pasteur 4lgérie, 1932, $10: 41-118$. 